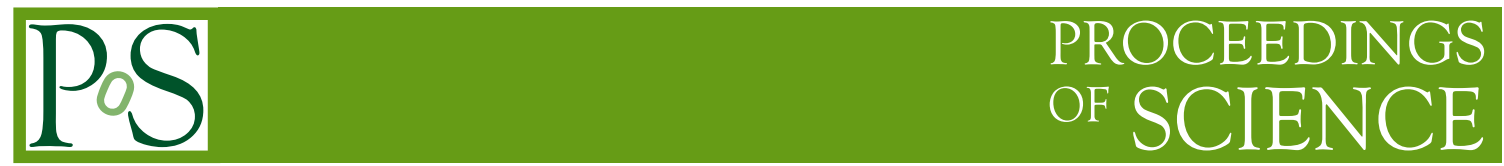

\title{
Hadronic structure from lattice QCD
}

\section{James M. Zanotti*}

School of Physics, University of Edinburgh, Edinburgh EH9 3JZ, UK

E-mail: jzanotti@ph.ed.ac.uk

Due to recent computer and algorithmic improvements, lattice calculations of hadronic quantities are now becoming available at pion masses as low as $m_{\pi} \approx 250 \mathrm{MeV}$. In this talk I review some of the latest lattice results for electromagnetic form factors of the nucleon, generalised parton distributions of the pion and nucleon, and distribution amplitudes of vector mesons and the nucleon.

LIGHT CONE 2008 Relativistic Nuclear and Particle Physics

July 7-11 2008

Mulhouse, France

${ }^{*}$ Speaker. 


\section{Introduction}

Lattice simulations provide a method for obtaining first principle results from QCD. However, one must have control of systematic effects such as finite volume, finite lattice spacing and large quark masses. This means that in order to compare with or provide predictions for experiment, we need to extrapolate the lattice results to the infinite volume and continuum limits, and the physical quark masses. This is an extremely difficult task, but with the advent of new computers, significant progress is now being made in getting these systematics under control [1].

In this talk I highlight the progress being made in lattice simulations in several areas of hadronic physics, including nucleon electromagnetic form factors in Sec. \& the nucleon isovector momentum fraction, $\langle x\rangle^{u-d}$, in Sec. 3, generalised parton distributions, including Ji's spin sum rule, impact parameter GPDs and transverse spin densities, in Sec. $甘$, and finally in Sec. E, I present some recent results of moments of vector and nucleon distribution amplitudes. For a comprehensive review of progress in the past year, see [2].

\section{Electromagnetic Form Factors}

On the lattice, we determine the form factors $F_{1}\left(q^{2}\right)$ and $F_{2}\left(q^{2}\right)$ by calculating the following matrix element of the electromagnetic current

$$
\left\langle p^{\prime}, s^{\prime}\left|j^{\mu}(\vec{q})\right| p, s\right\rangle=\bar{u}\left(p^{\prime}, s^{\prime}\right)\left[\gamma^{\mu} F_{1}\left(q^{2}\right)+i \sigma^{\mu v} \frac{q_{v}}{2 m_{N}} F_{2}\left(q^{2}\right)\right] u(p, s),
$$

where $u(p, s)$ is a Dirac spinor with momentum, $p$, and spin polarisation, $s, q=p^{\prime}-p$ is the momentum transfer, $m_{N}$ is the nucleon mass and $j_{\mu}$ is the electromagnetic current. The Dirac $\left(F_{1}\right)$ and Pauli $\left(F_{2}\right)$ form factors of the proton are obtained by using $j_{\mu}^{(p)}=\frac{2}{3} \bar{u} \gamma_{\mu} u-\frac{1}{3} \bar{d} \gamma_{\mu} d$, while for isovector form factors $j_{\mu}^{v}=\bar{u} \gamma_{\mu} u-\bar{d} \gamma_{\mu} d$. It is common to rewrite the form factors $F_{1}$ and $F_{2}$ in terms of the electric and magnetic Sachs form factors, $G_{e}=F_{1}+q^{2} /\left(2 m_{N}\right)^{2} F_{2}$ and $G_{m}=F_{1}+F_{2}$.

If one is using a conserved current, then (e.g. for the proton) $F_{1}^{(p)}(0)=G_{e}^{(p)}(0)=1$ gives the electric charge, while $G_{m}^{(p)}(0)=\mu^{(p)}=1+\kappa^{(p)}$ gives the magnetic moment, where $F_{2}^{(p)}(0)=\kappa^{(p)}$ is the anomalous magnetic moment. From Eq. (2.1) with see that $F_{2}$ always appears with a factor of $q$, so it is not possible to extract a value for $F_{2}$ at $q^{2}=0$ directly from our lattice simulations. Hence we are required to extrapolate the results we obtain at finite $q^{2}$ to $q^{2}=0$. Form factor radii, $r_{i}=\sqrt{\left\langle r_{i}^{2}\right\rangle}$, are defined from the slope of the form factor at $q^{2}=0$.

In Fig. 1 we see results for the isovector Dirac radius from several different fermion actions, including latest results from the RBC/UKQCD [3] (red circles) and LHP [ [ 4 ] (green right and black upside-down triangles) collaborations. These latest results are compared with earlier quenched and $N_{f}=2$ Wilson [5, 6] and domain-wall fermions (DWF) [7, 8] results. We observe agreement between the different lattice formulations, while any discrepancies are an indication for systematic uncertainties, such as finite volume effects, discretisation errors, etc. The overall pattern is typical of lattice results for $r_{1}$, i.e. the lattice results lie below experiment with little variation as a function of $m_{\pi}^{2}$. Investigations using chiral perturbation theory predict that these radii should increase dramatically close to the chiral limit [5, 9]. Current results indicate that in order to see such curvature, one needs to perform simulations at $m_{\pi}<300 \mathrm{MeV}$. 


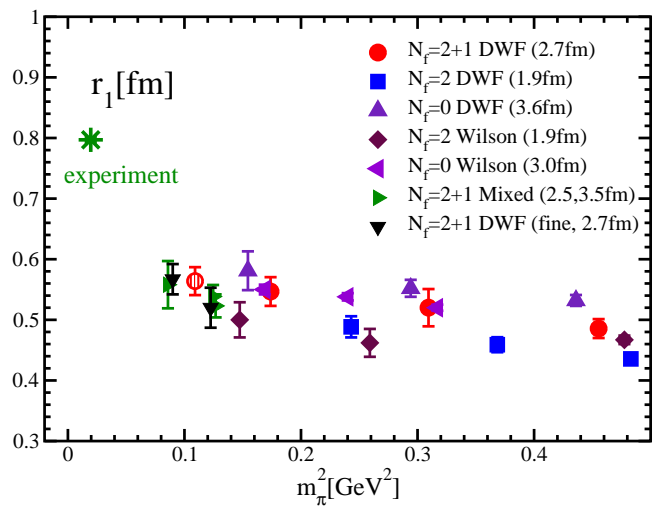

Figure 1: Comparison of results for the isovector Dirac radius, $r_{1}$.

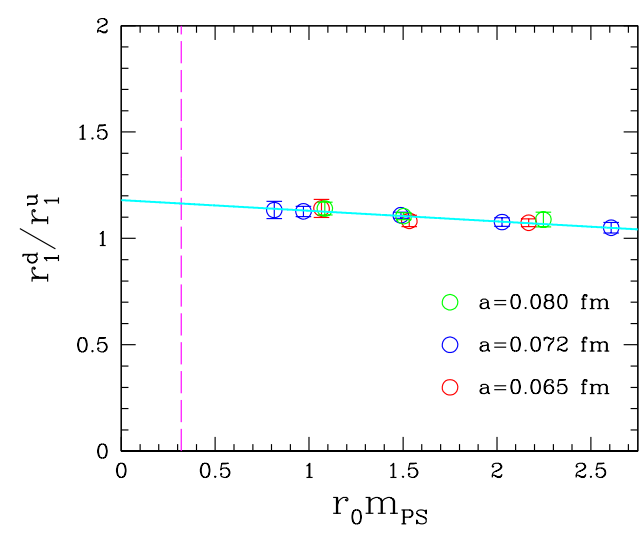

Figure 2: Ratio of Dirac radii for $u$ and $d$-quarks from QCDSF. Dashed line indicates physical $m_{\pi}$.

Lattice simulations of three-point functions are performed at the quark level, and hence they have an advantage over experiment in that they can directly measure the individual quark contributions to the nucleon form factors. Such simulations can provide insights into the internal distribution of charge and magnetisation in the nucleon. QCDSF have been studing the $q^{2}$-dependence of the individual quark contributions to the nucleon form factors, and in Fig. 2 we see some results for the ratio of the $d$ - to $u$-quark contributions to the proton's Dirac radius. Here we clearly see that $r_{1}^{d}>r_{1}^{u}$ for all simulated quark masses (the same behaviour is seen for $r_{2}$ ), indicating that the $d(u)$-quarks are more broadly distributed than $u(d)$-quarks in the proton (neutron). Note that contributions from disconnected diagrams were not considered in this study.

\subsection{Accessing small $Q^{2}$ : Partially twisted boundary conditions}

On a lattice of spatial size, $L$, momenta are discretised in units of $2 \pi / L$. Modifying the boundary conditions of the valence quarks [10] $\psi\left(x_{k}+L\right)=e^{i \theta_{k}} \psi\left(x_{k}\right),(k=1,2,3)$ allows one to tune the momenta continuously $\vec{p}+\vec{\theta} / L$. Momentum transfer in a matrix element between states with initial and final momenta, $\vec{p}_{i}+\vec{\theta}_{i} / L$ and $\vec{p}_{f}+\vec{\theta}_{f} / L$, respectively, then reads $q^{2}=\left(p_{f}-p_{i}\right)^{2}=$ $\left\{\left[E_{f}\left(\vec{p}_{f}, \vec{\theta}_{f}\right)-E_{i}\left(\vec{p}_{i}, \vec{\theta}_{i}\right)\right]^{2}-\left[\left(\vec{p}_{f}+\vec{\theta}_{f} / L\right)-\left(\vec{p}_{i}+\vec{\theta}_{i} / L\right)\right]^{2}\right\}$, where $E(\vec{p}, \vec{\theta})=\sqrt{m^{2}+(\vec{p}+\vec{\theta} / L)^{2}}$.

$F_{2}$ is particularly interesting since it cannot be measured directly at $q^{2}=0$ to obtain magnetic moments. Hence it needs to be extrapolated from finite $q^{2}$ which can not only increase the error, but can also introduce a model dependence into the result. Using partially twisted bc's to access small $q^{2}$ values can help to constrain the extrapolation to $q^{2}=0$ [11]. Additional $q^{2}$, s also allows us to try to fit the lattice data with more advanced fit forms, such as that proposed by Kelly [12]. QCDSF has attempted to use a form motivated by [12]

$$
F\left(Q^{2}\right)=F(0)\left(1+c_{1} Q^{2}+c_{n} Q^{2 n}\right)^{-1} .
$$

Fits using (2.2) to lattice data of $F_{1}^{n}\left(Q^{2}\right)$ and $G_{e}^{n}\left(Q^{2}\right)$ obtained with twisted bc's are shown in Fig. 3. Here we clearly see that the $F_{1}$ form factor of the neutron is negative at small $Q^{2}$. For $G_{e}^{n}$, we see a hump at small $Q^{2}$, similar but smaller than that seen in experimental data. It will be interesting to see how the "hump" behaves as we decrease the quark mass. 

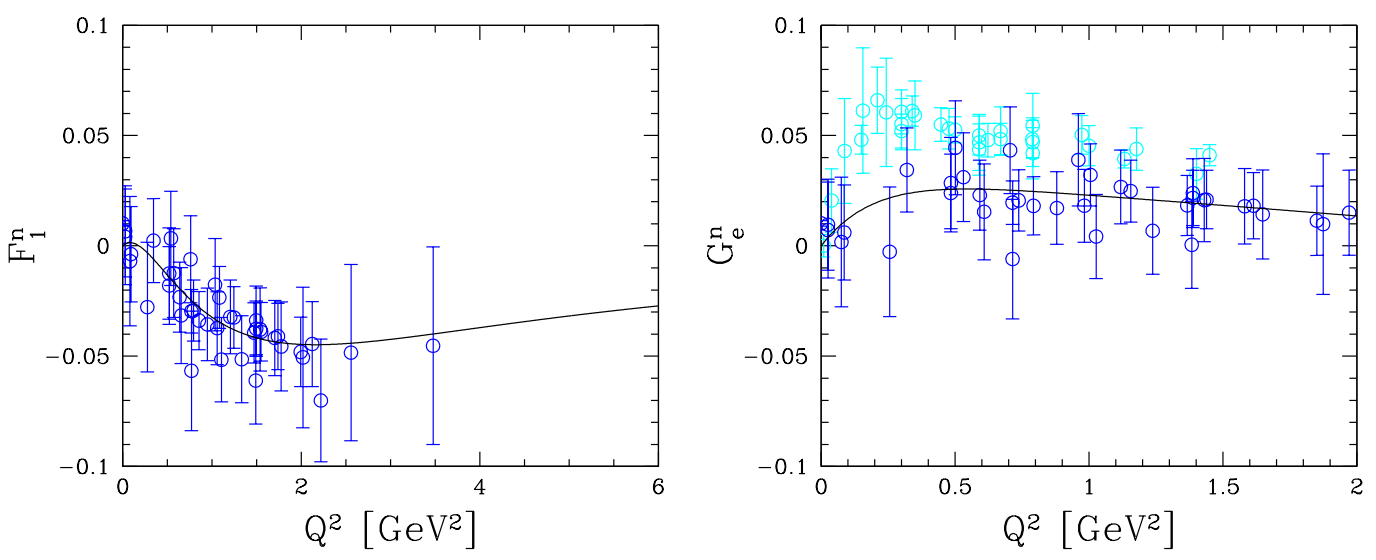

Figure 3: $F_{1}^{n}\left(Q^{2}\right)$ and $G_{e}^{n}\left(Q^{2}\right)$ using twisted boundary conditions and a fit in (2.2). Experimental data for $G_{e}^{n}$ are denoted by the lighter (cyan) points in the right plot.

\section{Nucleon Momentum Fraction, $\langle x\rangle$}

Lattice studies of $\langle x\rangle_{q}$ are notorious in that all lattice results to date at heavy quark masses exhibit an almost constant behaviour in quark mass towards the chiral limit and are almost a factor of two larger than phenomenologically accepted results, leading many a lattice practitioner to scratch their head and wonder "Will this thing ever bend down?", as predicted in [13].

To date, only connected contributions have been simulated to high precision, hence results are usually quoted for isovector quantities where disconnected contributions cancel.

Dynamical configurations are now becoming available at quark masses light enough to enable calculations in the area where such bending is predicted to set in. Latest results in the $\overline{\mathrm{MS}}$ scheme at $2 \mathrm{GeV}$ are shown in Fig. 7 from the QCDSF [14], LHP [15] and RBC/UKQCD [3] collaborations. In this figure we see excellent agreement between the older quenched [16, 17] and the latest $N_{f}=2+1$ DWF and $N_{f}=2$ clover results, with the possible exception of the lightest clover mass. This discrepancy may be attributed to a finite size effect $\left(m_{\pi} L=2.78\right)$, since these effects are expected to enhance $\langle x\rangle$ at light masses [18].

While we see agreement between the DWF

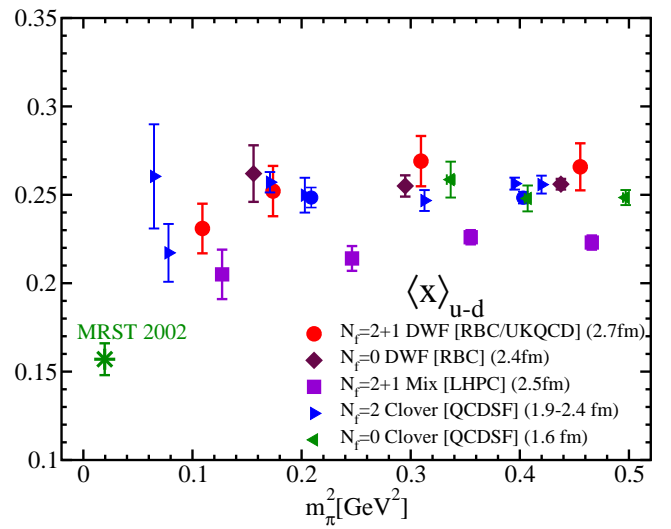

Figure 4: $\langle x\rangle_{u-d}$ from RBC/UKQCD (DWF), QCDSF (Clover) and LHPC (Mixed) and Clover results, we observe a gap between these results and those coming from the mixed action approach. Since the overall pion mass dependence is similar, this suggests that it is a renormalisation effect; a suggestion further enhanced when we consider that the results from the mixed approach use (non-perturbatively improved) perturbative renormalisation [15], while those from the other approaches use nonperturbative renor- 
malisation of the operators involved. Of course, for this issue to be fully resolved, the mixed action results need to be renormalised nonperturbatively.

\section{Generalised Parton Distributions}

A number of recent lattice results on moments of GPDs have been presented by QCDSF/UKQCD for the nucleon [19, 20] and the pion [21] and by LHPC for the nucleon [15]. Below, we discuss only a small selection lattice of these results, related to the nucleon and pion spin structure.

\subsection{Spin Sum Rules}

The total spin of the nucleon can be decomposed in terms of the quark and gluon angular momentum $1 / 2=\sum_{q} J_{q}\left(\mu^{2}\right)+J_{g}\left(\mu^{2}\right)$, which is then further decomposed into the quark and gluon spin and orbital angular momentum contributions $\frac{1}{2}=\sum_{q} \frac{1}{2} \Delta \Sigma_{q}+\sum_{q} L_{q}+\Delta G+L_{g}$, where $\Delta \Sigma$ and $\Delta G$ are the standard gauge-invariant quark and gluon spin fractions, while the orbital angular momentum contributions are defined by $L_{q}=J_{q}-\Delta \Sigma / 2$ and $L_{g}=J_{g}-\Delta G$. The gaugeinvariant total angular momenta $J_{q, g}$ are related to the forward values of the $(n=2)$-moments of the GPDs $H$ and $E$ through $2 J_{q, g}=\int d x x(H(x, \xi, 0)+E(x, \xi, 0))=\left(A_{20}^{q, g}(0)+B_{20}^{q, g}(0)\right)$ [22]. Since $A_{20}^{q, g}(0)=\langle x\rangle^{q, g}$ are simply the quark and gluon momentum fractions, we have by momentum conservation $1=\sum_{q} A_{20}^{q}(0)+A_{20}^{g}(0)$, hence we have a sum rule for the anomalous gravitomagnetic moments $0=\sum_{q} B_{20}^{q}(0)+B_{20}^{g}(0)$. Here we stress that although the sum is scale and scheme independent and is equal to zero, this is not the case for the individual $B_{20}^{q, g}$.

Figure 5 shows preliminary results from QCDSF/UKQCD for the nucleon spin decomposition in the $\overline{\mathrm{MS}}$ scheme at a scale of $4 \mathrm{GeV}^{2}[19$. It is remarkable that the $u+d$-quark OAM contribution is compatible with zero over the full range of accessible pion masses. The same behaviour has been observed by LHPC [15].

A potential improvement in the determination of $B_{20}^{q}$ from the lattice is in the extrapolation that is required from the simulated points at $q^{2} \neq 0$ to the required point $q^{2}=0$. As seen in Sec. 2.1, this can be achieved through the use of twisted boundary conditions, which is currently being ex-

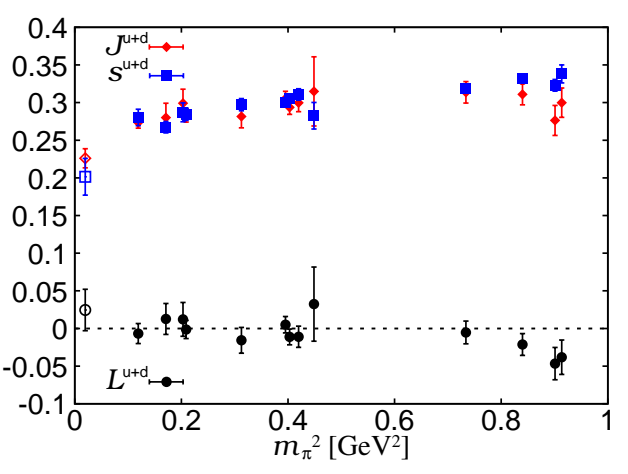

Figure 5: Decomposition of the nucleon spin from QCDSF/UKQCD [19] plored by the QCDSF collaboration [11]. This may become particularly important at light quark masses when the data becomes noisier, and hence the extrapolation is poorly constrained.

\subsection{Spin Asymmetries}

In the past couple of years, QCDSF have been computing the first two moments of vector and tensor GPDs in order gain an understanding on how quarks are spatially distributed inside the nucleon [20] and pion [21]. Of particular interest is the strong correlation between the transverse spin and coordinate degrees of freedom [23]. 
Impact densities of quarks with longitudinal momentum fraction, $x$, transverse spin, $s_{\perp}$, at a transverse distance, $b_{\perp}$, from the centre of momentum of a pion $\left(\rho\left(x, b_{\perp}, s_{\perp}\right)\right)$ or nucleon with transverse spin, $S_{\perp},\left(\rho\left(x, b_{\perp}, S_{\perp}, s_{\perp}\right)\right)$, can be directly obtained from the Impact Parameter GPDs, $H\left(x, b_{\perp}^{2}\right), E_{T}\left(x, b_{\perp}^{2}\right)$, etc.

The lowest two moments of these GPDs have been calculated recently by QCDSF for the nucleon [20] and pion [21]. In Fig. 6 we show the lowest $x$-moment of the density, $\rho^{n=1}\left(b_{\perp}, s_{\perp}\right)$ for up quarks in a $\pi^{+}$. Compared to the unpolarized case on the left, the density of quarks with transverse spin in $x$ direction on the right in Fig. 6is strongly distorted in the $b_{y}$-direction. A similar picture emerged from an analysis of nucleon GPDs [20] and proves the presence of strong cor-
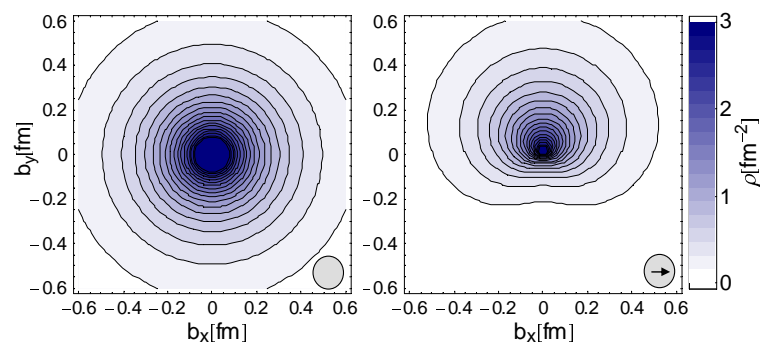

Figure 6: Densities of up-quarks in the $\pi^{+}$from QCDSF/UKQCD [21]. The arrow indicates the orientation of the transverse quark spin $s_{\perp}$. relations of the form $s_{\perp} \times b_{\perp}$ between trans-

verse spin and coordinate degrees of freedom of quarks in the pion. This distortion suggests [23] that the so-called Boer-Mulders function $h_{1}^{\pi, \perp}\left(x, k_{\perp}\right)$ of the pion is large and negative.

\section{Distribution Amplitudes}

Results for moments of the light pseudoscalar meson distribution amplitudes (DAs) have been presented by QCDSF [24] and UKQCD/RBC [25] in the last couple of years. Here we will focus on some recent results for vector mesons and the nucleon.

\subsection{Vector Mesons}

For spin-1 mesons, there are two twist-2 DA's, $\phi^{\|}(\xi), \phi^{\perp}(\xi)$, as opposed to a single DA for spin-0 mesons. The lowest moments of $\phi^{\|}(\xi)$ are obtained from the local matrix elements

$$
\begin{aligned}
\left\langle 0\left|\bar{q}(0) \gamma_{\{\rho} \stackrel{\leftrightarrow}{D}_{\mu\}} s(0)\right| V(p, \lambda)\right\rangle & =m_{V} f_{V} p_{\{\rho} \varepsilon_{\mu\}}^{(\lambda)}\left\langle\xi^{1}\right\rangle_{V}^{\|}, \\
\left\langle 0\left|\bar{q}(0) \gamma_{\{\rho} \stackrel{\leftrightarrow}{D}_{\mu} \stackrel{\leftrightarrow}{D}_{v\}} q(0)\right| V(p, \lambda)\right\rangle & =-i m_{V} f_{V} p_{\{\rho} p_{\mu} \varepsilon_{v\}}^{(\lambda)}\left\langle\xi^{2}\right\rangle_{V}^{\|},
\end{aligned}
$$

where $m_{V}$ and $f_{V}$ are the mass and decay constant, respectively, of the the vector meson, $V$, and $\varepsilon_{\mu}$ is a polarisation vector. The moments, $\left\langle\xi^{n}\right\rangle_{V}^{\|}$are extracted by constructing ratios of lattice two-point functions [26, 27] and the bare lattice results are then renormalised.

In Fig. 目 we see some preliminary results from the RBC/UKQCD collaborations for $\left\langle\xi^{1}\right\rangle_{K^{*}}^{\|}$ and $\left\langle\xi^{2}\right\rangle_{\rho}^{\|}$calculated with $N_{f}=2+1$ DWF configurations with 4 values of the light quark mass and 2 volumes [27]. The results indicate that there are no clear signs of finite volume effects. After renormalising to the $\overline{\mathrm{MS}}$ scheme at $\mu^{2}=4 \mathrm{GeV}^{2}$, they find

$$
\langle\xi\rangle_{K^{*}}^{\|} \approx 0.0359(17)(22) \quad\left\langle\xi^{2}\right\rangle_{\rho}^{\|} \approx 0.240(36)(12) \quad\left\langle\xi^{2}\right\rangle_{K^{*}}^{\|} \approx 0.252(17)(12)
$$

which compare well with the preliminary results from QCDSF [26]

$$
\langle\xi\rangle_{K^{*}}^{\|} \approx 0.036(3),\langle\xi\rangle_{K^{*}}^{\perp} \approx 0.030(2) .
$$



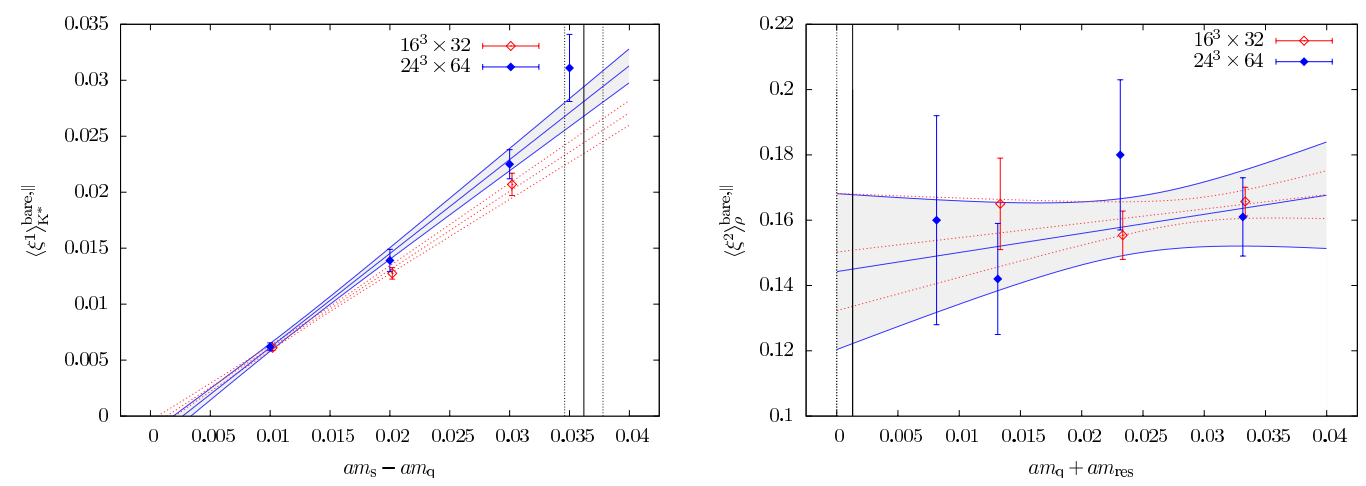

Figure 7: Unrenormalised results for $\left\langle\xi^{1}\right\rangle_{K^{*}}^{\|}$and $\left\langle\xi^{2}\right\rangle_{\rho}^{\|}$using $N_{f}=2+1$ Domain Wall Fermions on two different volumes [27].
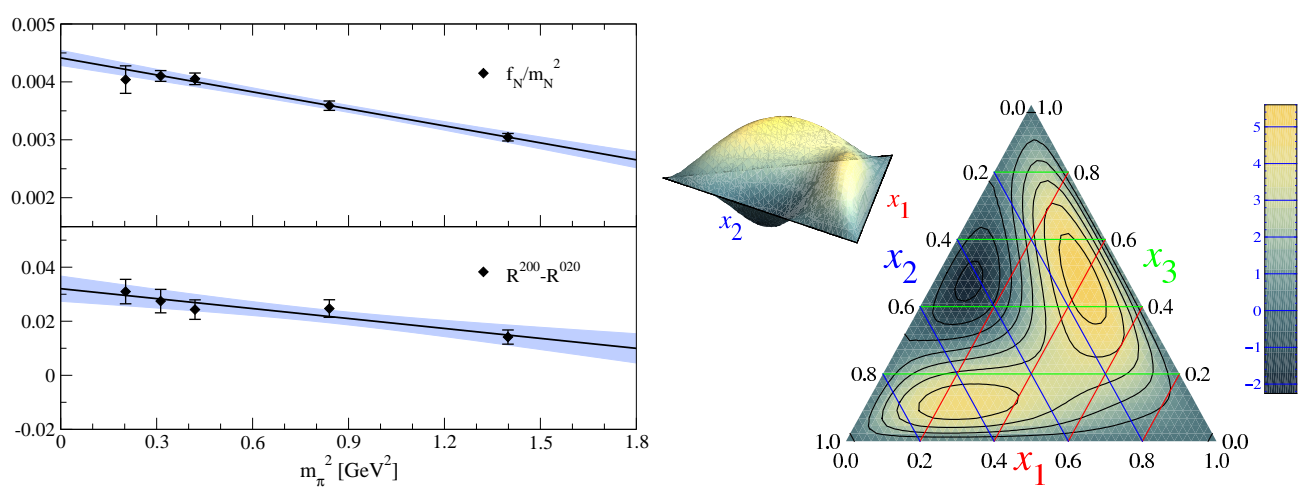

Figure 8: Nucleon decay constant, $f_{N}$, (top/left) and difference between ratios of moments (bottom/left) indicating an asymmetry between $\phi^{200}$ and $\phi^{020}$. Right: Barycentric contour plot of the leading-twist nucleon distribution amplitude. All results are in the $\overline{\mathrm{MS}}$ scheme at $2 \mathrm{GeV}$.

These results show the $S U(3)_{f}$-breaking effects in the $K^{*}$ DAs in a similar way to that observed for the $K$ DAs in [24, 25].

\subsection{Nucleon}

For the proton, there are three twist-2 distribution amplitudes, $V, A, T$. In a similar way to the case of mesons above, their moments $\left(V^{l m n}, A^{l m n}, T^{l n m}\right)$ can be obtained from hadronto-vacuum matrix elements of local operators [28]. It is useful to construct the combination, $\phi^{l m n}=\frac{1}{3}\left(V^{l m n}-A^{l m n}+2 T^{l n m}\right)$. In the asymptotic limit, $\varphi\left(x_{i}, Q^{2} \rightarrow \infty\right)=120 x_{1} x_{2} x_{3}$ and we have $\phi^{100}=\phi^{010}=\phi^{001}=\frac{1}{3}, \phi^{200}=\phi^{020}=\phi^{002}=\frac{1}{7}, \phi^{110}=\phi^{101}=\phi^{011}=\frac{2}{21}$, hence it is useful to look for asymmetries, such as $\phi^{100}-\phi^{010}$.

QCDSF have calculated first two moments [29] using an improved constrained analysis which considers ratios of correlators together with nonperturbative renormalisation of the appropriate 3quark operators [30]. By considering the difference between two such ratios, as shown in Fig. 8, the asymmetry is pronounced and increases as one approaches the chiral limit. 
These asymmetries are visualised in the right plot of Fig. 8, where the lattice moments have been used in a polynomial expansion of the full nucleon DA. Here $x_{1,2,3}$ refer to momentum fractions of the three quarks in the proton and the asymmetries indicate that the $u$-quark with spin aligned with the proton spin has largest momentum fraction $\left(x_{1}\right)$. Interestingly, the asymmetries are less pronounced than for QCD sum rules [31] and other phenomenological determinations [32].

\section{Conclusions}

Recently there has been an impressive amount of progress in lattice calculations of many different hadronic quantities, providing fascinating insights into the structure of hadrons. We have seen that the $Q^{2}$ scaling of hadronic form factors is now receiving an increasing amount of attention. In particular, twisted boundary conditions are providing access to small $Q^{2}$, but there also is work underway to attempt to probe the large $Q^{2}$ region $\left(>4 \mathrm{GeV}^{2}\right.$ ). The small $Q^{2}$ region is also an interesting place to study the Dirac and Sachs electric form factors of the neutron. The results that are now becoming available at small $Q^{2}$ are not only able to help constrain static quantities such as charge radii and magnetic/quadrupole moments, but also the value of the generalised form factor $B_{20}\left(q^{2}\right)$, which at $q^{2}=0$ provides the value of the anomalous gravitomagnetic moment, which is important in Ji's angular momentum sum rule.

While there appears to be a slight tension between the renormalisation of some of the lattice results for $\langle x\rangle_{u-d}$, the overall pattern seems to indicate that we may now at last be entering the region where the results may start to "bend down" towards the phenomenological value. However once again, FSE are predicted to become an issue close to the physical pion mass, so care will need to be taken to ensure this encouraging behaviour continues.

Lattice calculations of moments of generalised parton distributions are providing insights into the different quark contributions to the nucleon's spin and angular momentum, and current results indicate $J_{u} \approx 46 \%, J_{d} \approx 0, L_{u+d} \approx 0$. These moments are also providing evidence for non-trivial transverse spin densities in the pion and nucleon.

Following the recent success of lattice calculations of the moments of the light pseudoscalar meson distribution amplitudes (DAs), there are now results becoming available for moments of vector meson and proton DAs. Results for the proton are providing evidence that asymmetries exist in the way the momentum of the nucleon is distributed amongst its constituent quarks, with the $u$-quark with its spin aligned to that of the proton carrying the most momentum. The results also indicate that the symmetries are less pronounced than in QCD sum-rules.

\section{Acknowledgements}

I would like to thank my colleagues in the QCDSF and RBC/UKQCD collaborations. I acknowledge the support received through the STFC Advanced Fellowship Programme under contract number PP/F009658/1.

\section{References}

[1] K.I. Ishikawa, PoS LAT2008 (2008) 013; K. Jansen, PoS LAT2008 (2008) 010 [arXiv:0810.5634 [hep-lat]]. 
[2] J. M. Zanotti, PoS LAT2008 (2008) 007.

[3] S. Ohta and T. Yamazaki [RBC/UKQCD] PoS LAT2008 (2008) 168 [arXiv:0810.0045 [hep-lat]].

[4] J. D. Bratt et al., PoS LAT2008 (2008) 141 [arXiv:0810.1933 [hep-lat]].

[5] M. Göckeler et al. [QCDSF], Phys. Rev. D 71 (2005) 034508 [arXiv:hep-lat/0303019].

[6] C. Alexandrou et al., Phys. Rev. D 74 (2006) 034508 [arXiv:hep-lat/0605017].

[7] S. Sasaki and T. Yamazaki, Phys. Rev. D 78 (2008) 014510 [arXiv:0709.3150 [hep-lat]].

[8] H. W. Lin et al., arXiv:0802.0863 [hep-lat].

[9] R. D. Young et al., Phys. Rev. D 71 (2005) 014001 [arXiv:hep-lat/0406001].

[10] C. T. Sachrajda and G. Villadoro, Phys. Lett. B 609 (2005) 73 [arXiv:hep-lat/0411033]; P. F. Bedaque and J. W. Chen, Phys. Lett. B 616 (2005) 208 [arXiv:hep-lat/0412023]; P. F. Bedaque, Phys. Lett. B $\mathbf{5 9 3}$ (2004) 82 [arXiv:nucl-th/0402051].

[11] Ph. Hägler et al., PoS LAT2008 (2008) 138.

[12] J. J. Kelly, Phys. Rev. C 70 (2004) 068202.

[13] W. Detmold et al., Phys. Rev. Lett. 87 (2001) 172001 [arXiv:hep-lat/0103006].

[14] D. Brömmel et al., arXiv:0804.4706 [hep-lat].

[15] Ph. Hägler et al. [LHPC], Phys. Rev. D 77 (2008) 094502 [arXiv:0705.4295 [hep-lat]].

[16] M. Göckeler et al. [QCDSF], Phys. Rev. D 71 (2005) 114511 [arXiv:hep-ph/0410187].

[17] K. Orginos, T. Blum and S. Ohta, Phys. Rev. D 73 (2006) 094503 [arXiv:hep-lat/0505024].

[18] W. Detmold and C. J. D. Lin, Phys. Rev. D 71 (2005) 054510 [arXiv:hep-lat/0501007].

[19] D. Brömmel et al. [QCDSF/UKQCD], PoS LAT2007 (2007) 158 [arXiv:0710.1534 [hep-lat]].

[20] M. Göckeler et al. [QCDSF], Phys. Rev. Lett. 98 (2007) 222001 [arXiv:hep-lat/0612032].

[21] D. Brömmel et al. [QCDSF], Phys. Rev. Lett. 101 (2008) 122001 [arXiv:0708.2249 [hep-lat]].

[22] X. D. Ji, Phys. Rev. Lett. 78 (1997) 610 [arXiv:hep-ph/9603249].

[23] M. Diehl and Ph. Hägler, Eur. Phys. J. C 44 (2005) 87 [arXiv:hep-ph/0504175]; M. Burkardt, Phys. Rev. D 72 (2005) 094020 [arXiv:hep-ph/0505189]; S. Meissner, A. Metz and K. Goeke, Phys. Rev. D 76 (2007) 034002 [arXiv:hep-ph/0703176].

[24] V. M. Braun et al., Phys. Rev. D 74 (2006) 074501 [arXiv:hep-lat/0606012].

[25] P. A. Boyle et al., [UKQCD], Phys. Lett. B 641 (2006) 67 [arXiv:hep-lat/0607018]; M. A. Donnellan et al. [UKQCD], PoS LAT2007 (2007) 369 [arXiv:0710.0869 [hep-lat]].

[26] V. M. Braun et al. [QCDSF/UKQCD], PoS LAT2007 (2007) 144 [arXiv:0711.2174 [hep-lat]].

[27] P. A. Boyle et al., PoS LAT2008 (2008) 165 [arXiv:0810.1669 [hep-lat]].

[28] T. Kaltenbrunner et al., Eur. Phys. J. C 55 (2008) 387 [arXiv:0801.3932 [hep-lat]].

[29] M. Göckeler et al., Phys. Rev. Lett. 101 (2008) 112002 [arXiv:0804.1877 [hep-lat]].

[30] M. Göckeler et al., arXiv:0810.3762 [hep-lat].

[31] I. D. King and C. T. Sachrajda, Nucl. Phys. B 279 (1987) 785.

[32] J. Bolz and P. Kroll, Z. Phys. A 356 (1996) 327 [arXiv:hep-ph/9603289]; V. M. Braun, A. Lenz and M. Wittmann, Phys. Rev. D 73 (2006) 094019 [arXiv:hep-ph/0604050]. 